\title{
Feature Extraction of Hyperspectral Images Based On LBP and RF Feature Extraction Techniques
}

\author{
Soumya .M1, Dony D'souza² \\ ${ }^{1}$ VTU Belgaum, M.tech in Digital Electronics and Communication, Mangalore Institute of Technology and Engineering, Moodabidri. \\ Mangalore, Karnataka, India \\ ${ }^{2}$ Assistant Professor. Department of ECE., Mangalore Institute of Technology and Engineering, Moodabidri. \\ Mangalore, Karnataka, India
}

\begin{abstract}
This project deals with the feature extraction of hyperspectral images using LBP and RF based feature extraction techniques. Nowadays hyperspectral images find a wide variety of applications in the field of agriculture, eye care, mineralogy, astronomy and chemical imaging. Since hyperspectral images collect and process information from the entire EM spectrum, these images give exhaustive spectral data about the behavior of the objects. This information is used to discriminate various landscapes. Due to the high dimensionality of hyperspectral images, it is difficult to process the hyperspectral images. Feature extraction techniques are used to eliminate the dimensionality problems and reduce the computational complexity. The performance of this method is analyzed by using the quality indexes called, overall accuracy, average accuracy and kappa.
\end{abstract}

Keywords: Local binary pattern,Recursive filtering,Support vector machine,Overall accuracy,Average accuracy and Kappa

\section{Introduction}

Hyperspectral imaging is a method which combines the digital imaging with spectroscopy. A sensor on a moving platform collects the radiation from the entire earth's surface. The hyperspectral sensing is important for earth monitoring. The images acquired by the satellite can be used in different applications. Some of the applications include earth resource tracking, agricultural crop prediction, supervising the urban development and climate, controlling of fire and flood

The difference between hyperspectral images and an ordinary image is that hyperspectral images give more information about a scene compared to an ordinary image. The hyperspectral imaging splits the spectrum into several bands. The above method of partitioning the images into several bands can also be done beyond the visible band. The spectrum of hyperspectral imaging covers a wide range of wavelength and also has maximum resolution.

Airborne Visible-Infrared Imaging Spectrometer (AVIRIS) is one of the hyperspectral satellite sensors which are capable of providing good quality hyperspectral images. Since hyperspectral images collect and process information from the entire EM spectrum, these images give exhaustive spectral data about the behavior of the objects. This information is used to discriminate various landscapes. Since the number of channels in hyperspectral images is high, the processing of it is difficult. The high dimensionality of the hyperspectral images also causes Hughes phenomenon which degrades the classification performance [1].So that generating efficient way to process hyperspectral images is now becoming a difficult task. These problems can be solved by feature selection and extraction techniques, which are the crucial techniques in hyperspectral image classification.

Different techniques are already introduced for the feature extraction of hyperspectral images. By Feature extraction techniques, it is possible to eliminate the high dimensionality problem of hyperspectral images.

A novel feature extraction technique is introduced in this proposed approach. Feature extraction is done based on the Recursive filtering (RF) and Local binary pattern (LBP) feature extraction techniques. It helps to increase the accuracy of classification of the proposed method. The extracted features are given to a classifier. The classifier output will be a classification map.

\section{Proposed Method}

In this proposed method, a simple yet quite powerful feature extraction method is introduced .The image fusion is combined with Recursive filtering (RF) and Local binary pattern (LBP) feature extraction techniques which increase the classification accuracy.

A brief description of this method is as follows 1) the hyperspectral image is partitioned into multiple subsets of adjacent hyperspectral bands.2) the bands in each subset are fused together by averaging.3) The fused bands are processed with transform domain recursive filtering to get the resulting features for classification.4) LBP of the extracted features is formed. Support vector machines (SVMs) are used here as the classifier. By using this method, the accuracy of the SVM classifier can be improved significantly.Fig.1.shows the block diagram of the proposed method. The steps involved in this method are given below,

\section{1) Band partitioning:}

The hyperspectral image is spectrally partitioned into $\mathrm{S}$ subsets of hyperspectral bands as follows,

$$
\begin{aligned}
\mathrm{P}^{\mathrm{s}} & =\left(\mathrm{X}_{\mathrm{S}} \ldots, \mathrm{X}_{\left.\mathbb{G s}+\left\lfloor\frac{\mathrm{p}}{s}\right\rfloor\right)}\right) \text { if } \mathrm{s}+\lfloor\mathrm{D} / \mathrm{S}\rfloor \leq \mathrm{D} \\
& =\left(\mathrm{X}_{\mathrm{s}}, \ldots, \mathrm{X}_{\mathrm{D}}\right), \text { if } \mathrm{s}+\lfloor\mathrm{D} / \mathrm{S}\rfloor>\mathrm{D}
\end{aligned}
$$




\section{International Journal of Science and Research (IJSR) \\ ISSN (Online): 2319-7064 \\ Index Copernicus Value (2013): 6.14 | Impact Factor (2015): 6.391}

Where $\mathrm{x}=\left(\mathrm{X}_{1}, \ldots, \mathrm{X}_{\mathrm{D}}\right) \in \mathrm{R}^{\mathrm{D} \times \mathrm{J}}$ denotes the original hyperspectral image with $\mathrm{D}$-dimensional feature vectors with $\mathrm{J}$ pixels, and $[\mathrm{D} / \mathrm{S}\rfloor$ represents the floor operation which calculates the largest integer not greater than $\mathrm{D} / \mathrm{S}$.

\section{2) Image Fusion:}

The adjacent bands in the $\mathrm{s}^{\text {th }}$ subset are fused by one of the most simple IF methods, i.e., the averaging method.

$$
\mathrm{Q}^{\mathrm{s}}=\frac{\sum_{\mathrm{i}=1}^{\mathrm{N}_{\mathrm{S}}} \mathrm{P}_{\mathrm{i}}^{\mathrm{S}}}{\mathrm{N}_{\mathrm{S}}}
$$

Wherse $P_{i}^{s}$ refers to the $i^{\text {th }}$ band of the $s^{\text {th }}$ subset and $N_{s}$ refers to the total number of bands in the $s^{\text {th }}$ subset.

\section{3) Recursive filtering:}

Transform domain recursive filtering is performed on each fused band to obtain the $\mathrm{s}^{\text {th }}$ feature,

$$
\mathrm{O}_{\mathrm{j}}^{\mathrm{s}}=\mathrm{RF}_{\overline{\mathrm{os}}, \delta \mathrm{r} \mathrm{r}}\left(\mathrm{Q}_{\mathrm{j}}^{\mathrm{s}}\right)
$$

Where RF represents the transform domain recursive filtering operation, $\delta$ s and $\delta \mathrm{r}$ are the spatial and range standard deviations of the filter.

\section{4) LBP Feature extraction}

LBP feature extraction is performed on IF images. It generates binary pattern for each pixel in the image.

\section{5) Classification}

The SVM classifier is used for the classification of features.

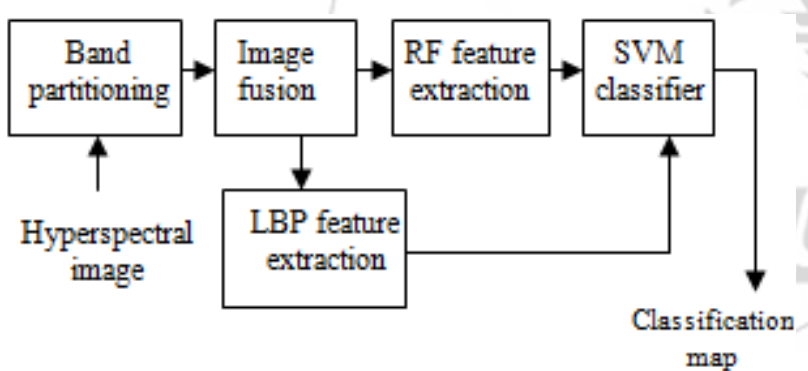

Figure 1: Simplified block diagram of the proposed method

\section{Experimental Result}

The Indian Pines image acquired by the AVIRIS sensor is used here as the input image. The image has 220 bands of size $145 \times 145$, with a spatial resolution of $20 \mathrm{~m}$ per pixel and a spectral coverage ranging from 0.4 to $2.5 \mu \mathrm{m}$.

Fig.2. Represents the input hyperspectral image. Fig.3 represents the corresponding output, the classification map.

\section{A) Quality indexes}

For the analysis of the performance, three quality indexes are used, average accuracy (AA), kappa and overall accuracy (OA).

$\mathrm{OA}$ is expressed in percentage which gives the number of correctly classified pixels. i.e., it measures the number of pixels which are same in the hyperspectral image and on ground truth. It indicates the total accuracy. AA represents the mean of total accuracy. It can be defined as the average of the percentage of correctly classified pixels. The percentage of correctly classified pixels corrected by the number of agreements that would be expected purely by chance is called kappa value.

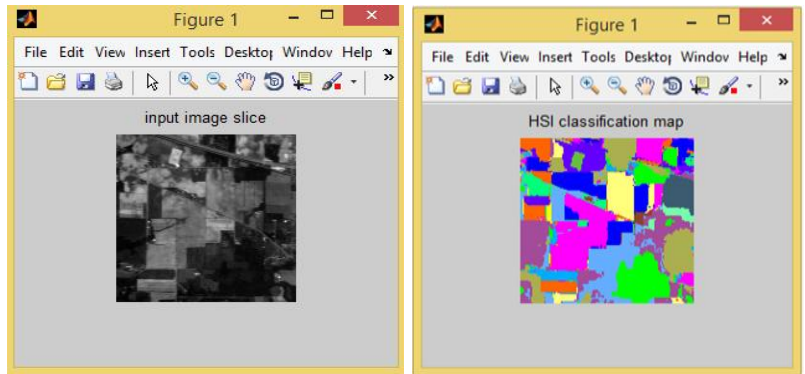

Figure 2:Input hyperspectral image Fig.3. Classification map

Table 1: Values of three quality indexes

\begin{tabular}{|c|c|}
\hline Quality index & Value \\
\hline OA & 0.9770 \\
\hline AA & 0.9770 \\
\hline KAPPA & 0.9734 \\
\hline
\end{tabular}

B) Filtered images with different spatial and range standard deviations

The values of range and spatial standard deviations of the filter have a significant role in the output of the filter. Let $\delta$ s denotes the spatial standard deviation and $\delta r$ indicates the range standard deviation. The Fig.4.shows how the filter output varies with different values of $\delta$ s and $\delta$.

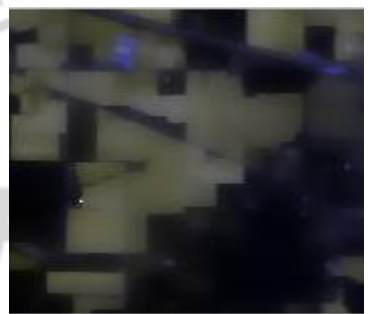

a) $\delta \mathrm{s}=6, \delta \mathrm{r}=0.3$

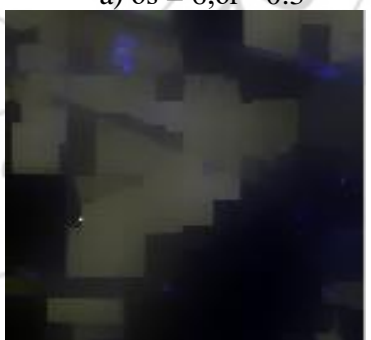

c) $\delta \mathrm{s}=60, \delta \mathrm{r}=0.3$

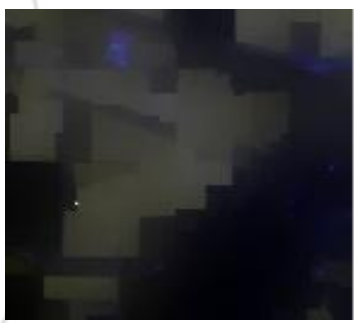

b) $\delta s=850, \delta r=0.3$

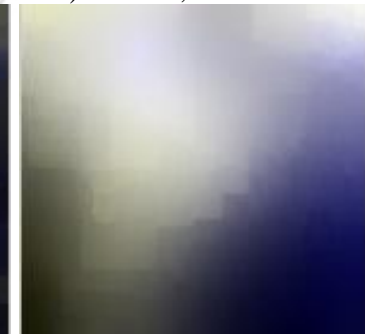

d) $\delta s=50, \delta r=2$

Figure 4: Filtered images with different spatial and range standard deviations

Observing the output images shown in Fig.4, It is clear that when the values of $\delta r$ and $\delta s$ are increased, it will smooth the filter outputs more evidently. Comparing with the other images, the value of $\delta r$ is high in Fig .4.d $(\delta r=2)$.In such case, the output of the filter is smoothed extremely. Which decreases the amount of valuable information and therefore only small amount of beneficial information is preserved. On the other hand when $\delta s$ increases and approaches infinity ( $\delta s=800$ in Fig.4. (b)) the recursive filter does not cause any smoothing effect on the image. 


\section{International Journal of Science and Research (IJSR) \\ ISSN (Online): 2319-7064}

Index Copernicus Value (2013): 6.14 | Impact Factor (2015): 6.391

\section{C) Comparison of feature extraction with EPF and the proposed method}

Three quality indexes of the proposed method is compared with the existing feature extraction method with Edge preserving filtering (EPF). The graph clearly shows that the proposed method has high performance compared to the existing method.

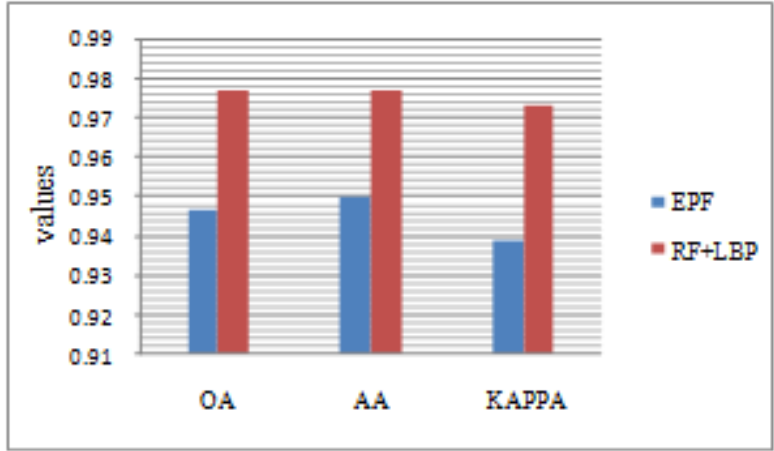

Figure 5: Comparison of feature extraction with EPF and the proposed method

\section{Conclusion}

Novel method to extract the features from a hyperspectral image is proposed. The proposed approach utilizes the advantages of image fusion techniques to reduce the difficulties related to the high dimensionality of the hyperspectral data. Both RF and LBP features of the input images are extracted. The performance of the method is measured by evaluating quality indexes. The results of this method indicate the effectiveness of the proposed approach. The attractive features of the proposed method are 1) it is time efficient and 2) the accuracy of the classification can be increased.

The future work of this approach includes the use of other EPFs for processing the fused images

\section{Acknowledgement}

I am very thankful to my guide Mr.DONY D'SOUZA, Assistant Professor, Department of Electronics and Communication Engineering, MITE for his cordial support, valuable information and constructive suggestions during the planning and development of this project work.

\section{References}

[1] G. Hughes, "On the mean accuracy of statistical pattern recognizers," IEEE Trans. Inf. Theory, vol. IT-14, no. 1, pp. 55-63, Jan. 1968.

[2] F. Melgani and L. Bruzzone, "Classification of hyperspectral remote sensing images with support vector machines," IEEE Trans. Geosci. Remote Sens., vol. 42, no. 8, pp. 1778-1790, Aug. 2004.

[3] X. Kang, S. Li, and J. A. Bendiktsson, "Spectral-spatial hyperspectral image classification with edge-preserving filtering," IEEE Trans. Geosci. Remote Sens., vol. 52, no. 5, pp. 2666-2677, May 2014.
[4] S. Prasad and L. Mann Bruce, "Limitations of principal components analysis for hyperspectral target recognition," IEEE Geosci. Remote Sens.Lett., vol. 5, no. 4, pp. 625629, Oct. 2008.

[5] Xudong Kang, and Jón Atli Benediktsson, "Feature Extraction of Hyperspectral Images With Image Fusion and Recursive Filtering" IEEE transactions on geoscience and remote sensing, vol. 52, no. 6, June 2014.

[6] J. Li, J. M. Bioucas-Dias, and A. Plaza, "Hyperspectral image segmentation using a new Bayesian approach with active learning," IEEE Trans. Geosci. Remote Sens., vol. 49, no. 10, pp. 3947-3960, Oct. 2011.

[7] W. Li, S. Prasad, J. E. Fowler, and L. Mann Bruce, "Locality-preserving discriminant analysis in kernelinduced feature spaces for hyperspectral image classification," IEEE Geosci. Remote Sens. Lett., vol. 8, no. 5, pp. 894-898, Sep. 2011.

[8] W. Li, S. Prasad, J. E. Fowler, and L. Mann Bruce, "Locality-preserving dimensionality reduction and classification for hyperspectral image analysis," IEEE Trans. Geosci. Remote Sens., vol. 50, no. 4, pp. 11851198, Apr. 2012.

[9] W. Liao, A. Pizurica, P. Scheunders, W. Philips, and Y. $\mathrm{Pi}$, "Semisupervised local discriminant analysis for feature extraction in hyperspectral images," IEEE Trans. Geosci. Remote Sens., vol. 51, no. 1, pp. 184-198, Jan. 2013

\section{Author Profile}

Soumya is pursuing M-tech in Digital electronics and communication branch, in Mangalore institute of technology and engineering Moodabidri, in the year of 2014-2016. 